\title{
Photon-assisted scattering and magnetoconductivity oscillations in a strongly correlated 2D electron system formed on the surface of liquid helium
}

\author{
Yu.P. Monarkha ${ }^{1}$ \\ ${ }^{1}$ Institute for Low Temperature Physics and Engineering, 47 Lenin Avenue, 61103 Kharkov, Ukraine
}

\begin{abstract}
The influence of strong internal forces on photon-assisted scattering and on the displacement mechanism of magnetoconductivity oscillations in a two-dimensional (2D) electron gas is theoretically studied. The theory is applied to the highly correlated system of surface electrons on liquid helium under conditions that the microwave frequency is substantially different from inter-subband resonance frequencies. A strong dependence of the amplitude of magnetoconductivity oscillations on the electron density is established. The possibility of experimental observation of such oscillations caused by photon-assisted scattering is discussed.
\end{abstract}

PACS numbers: 73.40.-c,73.20.-r,73.25.+i, 78.70.Gq

\section{INTRODUCTION}

Radiation-induced magnetoresistivity oscillations and zero resistance states (ZRS) of a $2 \mathrm{D}$ electron gas subjected to a perpendicular magnetic field were discovered in 2001-2003 using high quality GaAs/AlGaAs heterostructures $1-\underline{3}$. Since then, a large number of theoretical mechanisms have been proposed to explain these magneto-oscillations $(\mathrm{MO})^{\underline{4}} \underline{-11}$. The ZRS appeared at high radiation power as a result of evolution of resistivity minima can be caused by a negative conductivity effect $\left(\sigma_{x x}<0\right)^{12}$, whose microscopic origin is quite controversial as well as the origin of MO.

In experiments $1-\underline{3}$, the microwave (MW) frequency was quite arbitrary: $\omega>\omega_{c}$, here $\omega_{c}$ is the cyclotron frequency. The period of MO observed is controlled by the ratio $\omega / \omega_{c}$. Similar $1 / B$-periodic oscillations of magnetoconductivity $\sigma_{x x}$ and ZRS were discovered in a nondegenerate $2 \mathrm{D}$ electron system formed on the free surface of liquid helium when the MW frequency was tuned to the inter-subband excitation frequency 13.14: $\omega=\omega_{2,1} \equiv$ $\left(\Delta_{2}-\Delta_{1}\right) / \hbar$ (here $\Delta_{l}$ is the energy spectrum of surface subbands, $l=1,2, \ldots)$. These oscillations were theoretically explained 15 by a nonequilibrium population of the excited subband and by peculiarities of quasielastic intersubband scattering in the presence of the magnetic field $B$. Predictions of this intersubband displacement model concerning the influence of internal Coulomb forces on positions of $\sigma_{x x}$ extrema $\frac{16}{6}$ were recently supported by experimental observations 17 .

For mechanisms of MO proposed ${ }^{4.5}, \underline{9}$, electron gas degeneracy is not a crucial point. Therefore, they can be applied also to a nondegenerate 2D electron system like surface electrons (SEs) on liquid helium. Electrons bound to the free surface of liquid helium represent a remarkable model 2D system which is quite simple and clean. SEs are scattered quasi-elastically by capillary wave quanta (ripplons) and by vapor atoms. Their interaction parameters are well established. Experiments on SEs $\frac{13,14}{}$ employed approximately the same MW frequencies and power as those used for the 2D electron gas in $\mathrm{GaAs} / \mathrm{AlGaAs}^{1} \stackrel{-3}{-3}$. Therefore, there is an impor- tant question: why theoretical mechanisms of $\mathrm{MO}$ and negative conductivity effects proposed for semiconductor electrons do not display themselves in experiments with SEs on liquid helium? The answer to this question could shed light also on the situation in semiconductor systems.

The most frequently discussed mechanism of MO and negative conductivity effects called the displacement mechanism was proposed already in 1969 by Ryzhii 18 . In this model a quasielastic scattering event of an electron caused by an impurity potential can be accompanied by absorption of a photon, which leads to indirect inter-Landau-level scattering $\left(n \rightarrow n^{\prime}\right)$. The energy conservation of such a photon-assisted scattering event

$$
\hbar \omega_{c}\left(n-n^{\prime}\right)+\hbar \omega+e E_{\|}\left(X-X^{\prime}\right)=0
$$

(here $E_{\|}$is the dc driving electric field directed along the $x$-axis) determines the displacement of the electron orbit center, which can be opposite to the driving force $\left(X^{\prime}>X\right)$ if $\omega / \omega_{c}<n^{\prime}-n$. This is the reason for the negative conductivity effect. It should be noted that in the intersubband displacement mode ${ }^{15,16}$ the corresponding energy conservation contains the intersubband excitation energy $\Delta_{2}-\Delta_{1}$ instead of $\hbar \omega$. The displacement mode118 had been intensively developed after discovery of ZRS to include self-energy effects and multiphoton processes $4,5,19$.

Another popular mechanism of MO and negative conductivity effects called the inelastic mode $\underline{1}^{\underline{9}}$ originates from oscillations of the electron distribution function $f(\varepsilon)$ induced by MW radiation. An oscillatory behavior of $f(\varepsilon)$ means that at certain ranges of $\varepsilon$ there is a population inversion, $\partial f(\varepsilon) / \partial \varepsilon>0$, and the magnetoconductivity $\sigma_{x x}$ could be negative. These ideas were also discussed in Ref. 20.

In this work, we consider mostly the displacement mechanism of MO applied to the system of SEs in liquid helium: photon-assisted scattering by ripplons. The inelastic mechanism will be shortly discussed as well, since we see its certain relation to photon-assisted scattering. As compared to the initial version of photon-assisted scattering 18,21 , the theory is extended to include strong Coulomb forces acting between electrons and the collision 
broadening of Landau levels (LLs). We found the reason why MO of $\sigma_{x x}$ and ZRS caused by photon-assisted scattering were not seen in experiments on $\mathrm{SEs}^{13,14}$ for chosen ranges of the MW frequency and amplitude, and formulated conditions under which they could be potentially observed.

\section{PROBABILITIES OF PHOTON-ASSISTED SCATTERING}

Consider a 2D electron gas on the free surface of liquid helium in the presence of a static magnetic field $\mathbf{B}$ directed normally to the interface. The corresponding vector potential of the magnetic field $\mathbf{A}_{0}=(0, B x, 0)$. In the presence of the driving electric field $\mathbf{E}_{\|}=\left(E_{\|}, 0,0\right)$, eigenfunctions and the energy spectrum of SEs are characterized by the surface subband number $l$, the electron orbit center

$$
X=-\frac{c p_{y}}{e B}+\zeta, \quad \zeta=-\frac{e E_{\|}}{m_{e} \omega_{c}^{2}},
$$

and by the LL number $n$ :

$$
\begin{aligned}
& \Psi_{l, n, X}=\psi_{l}(z) \frac{1}{\sqrt{2 \pi \hbar}} e^{-i(X-\zeta) y / l_{B}^{2}} \psi_{n}^{(\mathrm{os})}(x-X), \\
& E_{l, n, X}=\Delta_{l}+\hbar \omega_{c}(n+1 / 2)+e E_{\|} X+\frac{e^{2} E_{\|}^{2}}{2 m_{e} \omega_{c}^{2}},
\end{aligned}
$$

Here $\psi_{n}^{(\mathrm{os})}(x)$ are oscillator eigenfunctions, $\psi_{l}(z)$ are wavefunctions of vertical motion, and $l_{B}=\sqrt{\hbar c / e B}$ is the magnetic length.

At low temperatures $T<1 \mathrm{~K}$, electrons predominantly occupy the ground surface subband $(l=1)$, since $\Delta_{2}-\Delta_{1}$ is about $6 \mathrm{~K}$ (liquid ${ }^{4} \mathrm{He}$ ) or $3.2 \mathrm{~K}$ (liquid ${ }^{3} \mathrm{He}$ ). The Hamiltonian of electron interaction with MWs has the usual form

$$
V_{m w}=\frac{e}{c m_{e}}\left(\mathbf{p}+\frac{e}{c} \mathbf{A}_{0}\right) \tilde{\mathbf{A}}
$$

where $\tilde{\mathbf{A}}$ is the vector potential of the MW field,

$$
\tilde{A}_{\alpha}(\mathbf{r})=\sum_{\lambda, \mathbf{k}}\left(\frac{2 \pi \hbar c^{2}}{\omega_{k} K_{\mathrm{v}}}\right)^{1 / 2} e_{\alpha, \lambda, \mathbf{k}} e^{i \mathbf{k} \cdot \mathbf{r}}\left(a_{\mathbf{k}, \lambda}+a_{-\mathbf{k}, \lambda}^{\dagger}\right)
$$

$a_{\mathbf{k}, \lambda}$ and $a_{\mathbf{k}, \lambda}^{\dagger}$ are creation and destruction operators of photons, $K_{\mathrm{v}}$ is the volume, and $\mathbf{e}_{\lambda, \mathbf{k}}$ is the unit polarization vector. It is convenient to consider the linear polarization with $\tilde{A}_{y}=0$.

The Hamiltonian of the electron-ripplon interaction, which dominates under conditions of the experiments $(T=0.2 \mathrm{~K})^{14}$, can be written as

$$
V_{r}=\sum_{\mathbf{q}} V_{r, q} Q_{q}\left(b_{\mathbf{q}}+b_{-\mathbf{q}}^{\dagger}\right) e^{i \mathbf{q} \cdot \mathbf{r}}
$$

where $b_{\mathbf{q}}$ and $b_{-\mathbf{q}}^{\dagger}$ are creation and destruction operators of ripplons, $V_{r, q}$ is the electron-ripplon coupling ${ }^{22}, Q_{q}=$ $\sqrt{\hbar q / 2 \rho \omega_{r, q} D}, \omega_{r, q} \simeq \sqrt{\alpha / \rho} q^{3 / 2}, \alpha$ and $\rho$ are the surface tension and mass density of liquid helium respectively, and $D$ is the surface area.

Probabilities of photon-assisted scattering are calculated according to the Golden Rule with the following matrix elements ${ }^{21}$

$$
\begin{gathered}
\left\langle i|\tilde{V}| f_{ \pm}\right\rangle=\sum_{v} \frac{\left\langle i\left|V_{r}\right| v\right\rangle\left\langle v\left|V_{m w}\right| f_{ \pm}\right\rangle}{E_{i}-E_{v}}+ \\
\quad+\sum_{v} \frac{\left\langle i\left|V_{m w}\right| v\right\rangle\left\langle v\left|V_{r}\right| f_{ \pm}\right\rangle}{E_{i}-E_{v}}
\end{gathered}
$$

where $E_{i}$ is the energy of the initial state, the final state $\left|f_{ \pm}\right\rangle=\left|n^{\prime}, X^{\prime}, \mathbf{n}_{\mathbf{q}}^{(r)} \pm \mathbf{1}_{ \pm \mathbf{q}}, \mathbf{n}_{\mathbf{k}}^{(m w)}-\mathbf{1}_{-\mathbf{k}},\right\rangle$ corresponds to processes of destruction of a photon and creation (sign plus) or destruction (sign minus) of a ripplon. The $\mathbf{n}_{\mathbf{q}}^{(r)}=\left\{n_{\mathbf{q}}^{(r)}\right\}$ and $\mathbf{n}_{\mathbf{k}}^{(m w)}=\left\{n_{\mathbf{k}}^{(m w)}\right\}$ are the vectors describing occupation numbers of ripplons and photons.

Further evaluations are based on the relationship $\left(e^{i \mathbf{q r}_{e}}\right)_{n, X ; n^{\prime}, X^{\prime}}=\delta_{X, X^{\prime}-l_{B}^{2} q_{y}} e^{i q_{x} X} M_{n, n^{\prime}}$ which is valid for the chosen gauge. Here we use the following notations

$$
\begin{gathered}
M_{n, n^{\prime}}\left(x_{q}, \varphi\right)=i^{\left|n^{\prime}-n\right|} J_{n, n^{\prime}}\left(x_{q}\right) \times \\
\times \exp \left[i x_{q} \cos (\varphi) \sin (\varphi)+i\left(n^{\prime}-n\right) \varphi\right], \\
J_{n, n^{\prime}}\left(x_{q}\right)=\sqrt{\frac{\min \left(n, n^{\prime}\right) !}{\max \left(n, n^{\prime}\right) !}} x_{q}^{\frac{\left|n^{\prime}-n\right|}{2}} \times \\
\times \exp \left(-\frac{x_{q}}{2}\right) L_{\min \left(n, n^{\prime}\right)}^{\left|n^{\prime}-n\right|}\left(x_{q}\right),
\end{gathered}
$$

where $x_{q}=q^{2} l_{B}^{2} / 2$ is a dimensionless parameter, $q_{x}=$ $q \cos \varphi$, and $L_{n}^{m}(x)$ are the associated Laguerre polynomials. Our expression for $M_{n, n^{\prime}}\left(x_{q}, \varphi\right)$ differs from the similar equation of Ref. 21 because we have chosen the different gauge. The result of Ref. 21 can be restored from Eq. (9) using the substitution $\varphi \rightarrow \varphi-\pi / 2$.

Introducing

$$
\mathcal{E}(\omega)=\sqrt{4 \pi \frac{n^{(m w)}(\omega)}{K_{\mathrm{v}}} \hbar \omega}
$$

and $C_{\mathbf{q}}^{( \pm)}=V_{r, q} Q_{q}\left[n_{ \pm \mathbf{q}}^{(r)}+\frac{1}{2} \pm \frac{1}{2}\right]^{1 / 2}$, and following the procedure described in Ref. 21, one can find

$$
\left|\left\langle i|\tilde{V}| f_{ \pm}\right\rangle\right|=C_{\mathbf{q}}^{( \pm)} \frac{e \mathcal{E}(\omega)}{2 m_{e} \omega^{2} l_{B}} \delta_{X, X^{\prime}-l_{B}^{2} q_{y}} \times
$$




$$
\sum_{n^{\prime \prime}}\left[\frac{\omega M_{n, n^{\prime \prime}} p_{n^{\prime \prime}, n^{\prime}}}{\omega_{c}\left(n-n^{\prime \prime}\right)-q_{y} V_{H} \mp \omega_{r, q}}+\frac{\omega p_{n, n^{\prime \prime}} M_{n^{\prime \prime}, n^{\prime}}}{\omega_{c}\left(n-n^{\prime \prime}\right)+\omega}\right],
$$

where $p_{n, n^{\prime}}=i\left(\sqrt{n} \delta_{n^{\prime}, n-1}-\sqrt{n^{\prime}} \delta_{n^{\prime}, n+1}\right)$ are dimensionless matrix elements of the electron momentum operator, and $V_{H}$ is the absolute value of the Hall velocity: $e E_{\|}\left(X-X^{\prime}\right)=-\hbar q_{y} V_{H}$.

Keeping in mind the energy conservation deltafunction, in the first term of Eq. (12) we can use the replacement $\omega_{c}\left(n-n^{\prime \prime}\right)-q_{y} V_{H} \mp \omega_{r, q} \rightarrow \omega_{c}\left(n^{\prime}-n^{\prime \prime}\right)-\omega$. For slightly broadened LLs, this is an approximate procedure which has the same accuracy as the replacement $1-f\left(\varepsilon^{\prime}\right) \rightarrow 1-f(\varepsilon)$ in usual conductivity equations. Then, using properties of the associated Laguerre polynomials, one can find

$$
\begin{aligned}
& \left|\left\langle i|\tilde{V}| f_{ \pm}\right\rangle\right|^{2}=\left(C_{\mathbf{q}}^{( \pm)}\right)^{2}\left[\frac{e \mathcal{E}(\omega)}{2 m_{e} \omega^{2} l_{B}}\right]^{2} \times \\
& \times \delta_{X, X^{\prime}-l_{B}^{2} q_{y}} \chi_{\mathbf{q}}(\omega) x_{q} J_{n, n^{\prime}}^{2}\left(x_{q}\right),
\end{aligned}
$$

where

$$
\chi_{\mathbf{q}}(\omega)=\left|\frac{e^{i \varphi} \omega}{\omega_{c}+\omega}-\frac{e^{-i \varphi} \omega}{\omega_{c}-\omega}\right|^{2} .
$$

The dependence of $\chi_{\mathbf{q}}$ on $\varphi$ is important for calculation of the momentum relaxation rate, which contains an additional factor $q_{y}^{2}=q^{2} \sin ^{2} \varphi$.

After summation over $\mathbf{k}$ the probabilities of photonassisted scattering from $n, X$ to $n^{\prime}, X^{\prime}$ accompanied by the momentum exchange $\hbar \mathbf{q}$ can be found as

$$
\begin{gathered}
w_{n, X \rightarrow n^{\prime}, X^{\prime}}^{( \pm)}(\mathbf{q})=\frac{2 \pi}{\hbar}\left(C_{\mathbf{q}}^{( \pm)}\right)^{2} \lambda_{m w} \chi_{\mathbf{q}}(\omega) x_{q} \times \\
\times \delta_{X, X^{\prime}-l_{B}^{2} q_{y}} J_{n, n^{\prime}}^{2}\left(x_{q}\right) \delta\left(\varepsilon_{n}-\varepsilon_{n^{\prime}}-\hbar q_{y} V_{H} \mp \hbar \omega_{q}+\hbar \omega\right), \\
\text { where } \varepsilon_{n}=\hbar \omega_{c}(n+1 / 2), \\
\lambda_{m w}=\frac{e^{2} E_{m w}^{2}}{4 m_{e}^{2} \omega^{4} l_{B}^{2}}, \quad E_{m w}^{2}=4 \pi \frac{N_{m w}(\omega)}{K_{\mathrm{v}}} \hbar \omega
\end{gathered}
$$

$N_{m w}(\omega)$ is the number of photons with the frequency $\omega$, and $E_{m w}\left(\sqrt{\mathrm{ergs} / \mathrm{cm}^{3}}\right)$ is the amplitude of the electric field in the MW. As compared to usual electron-ripplon scattering, Eq. (15) contains the photon energy $\hbar \omega$ in the argument of the delta-function and additional dimensionless proportionality factors $\lambda_{m w}, \chi_{\mathbf{q}}(\omega)$, and $x_{q}$. The $\chi_{\mathbf{q}}(\omega)$ is of the order of unity (here $\omega$ is substantially larger than $\omega_{c}$ ), while $x_{q}$ is of the order of $n^{\prime}-n$ due to $J_{n, n^{\prime}}^{2}\left(x_{q}\right)$. The $\lambda_{m w}$ depends only on the MW field parameters $\left(E_{m w}\right.$ and $\left.\omega\right)$ and on basic properties of the electron gas under magnetic field.

\section{THE CONDUCTIVITY OF STRONGLY INTERACTING ELECTRONS}

Generally, the structure of Eq. (15) is similar to the structure of the corresponding probability of the usual electron-ripplon scattering. Therefore, considering the contribution of photon-assisted scattering into the momentum relaxation rate, we can use advantages of the approach $^{22}-24$, which allows to express average scattering probabilities and the momentum relaxation rate $\nu_{\text {eff }}$ in terms of the dynamic structure factor (DSF) of the $2 \mathrm{D}$ electron system. Since $\sum_{X^{\prime}} w_{n, X \rightarrow n^{\prime}, X^{\prime}}^{( \pm)}(\mathbf{q})$ does not depend on $X$, when averaging over the initial electron states we can consider electron distribution over LLs only $f_{n} \simeq Z_{\|}^{-1} e^{-\varepsilon_{n} / T_{e}}$ (here $Z_{\|}=\sum_{n} e^{-\varepsilon_{n} / T_{e}}$ ). Then, the average probability of electron scattering with the momentum exchange $\hbar \mathbf{q}$ caused by destruction of a photon and creation or destruction of a ripplon can be written in the following form

$$
\begin{gathered}
\bar{w}_{\mathrm{mw}, \mathbf{q}}^{( \pm)}=\frac{1}{\hbar^{2}}\left(C_{r, \mathbf{q}}^{( \pm)}\right)^{2} \lambda_{m w} \chi_{\mathbf{q}}(\omega) \times \\
\times x_{q} S\left(q,-q_{y} V_{H} \mp \omega_{r, q}+\omega\right),
\end{gathered}
$$

where $C_{r, \mathbf{q}}^{( \pm)}$is obtained from $C_{\mathbf{q}}^{( \pm)}$replacing the ripplon occupation number $n_{ \pm \mathbf{q}}^{(r)}$ with the Bose distribution function $N_{r, \pm \mathbf{q}}$, the function

$$
\begin{aligned}
& S(q, \Omega)=\frac{2}{\pi \hbar Z_{\|}} \sum_{n, n^{\prime}} J_{n, n^{\prime}}^{2}\left(x_{q}\right) \times \\
& \times \int d \varepsilon e^{-\varepsilon / T_{e}} g_{n}(\varepsilon) g_{n^{\prime}}(\varepsilon+\hbar \Omega)
\end{aligned}
$$

is the DSF of a nondegenerate 2D electron gas, $g_{n}(\varepsilon)=$ $-\operatorname{Im} G_{n}(\varepsilon)$, and $G_{n}(\varepsilon)$ is the single-electron Green's function.

Taking into account the collision broadening of LLs, we shall use the result of the cumulant approach 25

$$
g_{n}(\varepsilon)=\frac{\sqrt{2 \pi} \hbar}{\Gamma_{n}} \exp \left[-\frac{2\left(\varepsilon-\varepsilon_{n}\right)^{2}}{\Gamma_{n}^{2}}\right],
$$

where $\Gamma_{n}$ is the collision broadening of LLs 26 . This approximation, being quite accurate for low LLs, greatly simplifies further evaluations. The equilibrium DSF has an important property

$$
S(q,-\Omega)=e^{-\hbar \Omega / T_{e}} S(q, \Omega)
$$

which allows to shorten evaluations. According to Eqs. (18) and (19), the $S(q, \Omega)$ as a function of frequency has sharp maxima when $\Omega$ approaches the LL excitation frequency $\left(n^{\prime}-n\right) \omega_{c}$.

The momentum relaxation rate can be found by evaluating the total momentum gained by scatterers. For an 
infinite isotropic system, the kinetic friction acting on the electron gas,

$$
\mathbf{F}_{\text {fric }}=-N_{e} \sum_{\mathbf{q}} \hbar \mathbf{q}\left(\bar{w}_{r, \mathbf{q}}+\bar{w}_{\mathrm{mw}, \mathbf{q}}^{(+)}+\bar{w}_{\mathrm{mw}, \mathbf{q}}^{(-)}\right),
$$

should be antiparallel to the current. Here $\bar{w}_{r, \mathbf{q}} \equiv$ $\bar{w}_{r, \mathbf{q}}^{(+)}+\bar{w}_{r, \mathbf{q}}^{(-)}$is the corresponding probability obtained for usual electron-ripplon scattering in the absence of MW radiation, and $\bar{w}_{r, \mathbf{q}}^{( \pm)}$can be found from Eq. (17) using the substitution $\lambda_{m w} \chi_{\mathbf{q}}(\omega) x_{q} \rightarrow 1$ and setting $\omega \rightarrow 0$ in the frequency argument of the DSF.

Thus, the momentum relaxation rate $\nu_{\text {eff }}$ can be defined by the relationship $\left(\mathbf{F}_{\text {fric }}\right)_{y}=-N_{e} m_{e} \nu_{\text {eff }} V_{y}$, where $V_{y} \simeq-V_{H}$. Using elastic approximation $\left(\hbar \omega_{r, q} \rightarrow 0\right)$, the correction into the effective collision frequency induced by MW radiation is found as

$$
\nu_{\mathrm{mw}}=\frac{2 \lambda_{m w}}{m_{e} \hbar} \sum_{\mathbf{q}} q_{y}^{2} \chi_{\mathbf{q}}(\omega) x_{q} C_{r, \mathbf{q}}^{2} S^{\prime}(q, \omega),
$$

where $C_{r, \mathbf{q}} \equiv C_{r, \mathbf{q}}^{(-)} \simeq C_{r, \mathbf{q}}^{(+)}$, and $S^{\prime}(q, \Omega)=$ $\partial S(q, \Omega) / \partial \Omega$.

In the absence of MW radiation, the momentum relaxation rate $\nu_{r}^{(0)}$ can be formally obtained from Eq. (22) using the replacement $\lambda_{m w} \chi_{\mathbf{q}}(\omega) x_{q} \rightarrow 1$ and setting $\omega \rightarrow 0$ in the frequency argument of $S^{\prime}$ :

$$
\nu_{r}^{(0)}=\frac{2}{m_{e} \hbar} \sum_{\mathbf{q}} q_{y}^{2} C_{r, \mathbf{q}}^{2} S^{\prime}(q, 0) .
$$

The derivative of the Eq. (20) gives the relationship

$$
S^{\prime}(q, 0)=\frac{\hbar}{2 T_{e}} S(q, 0)>0,
$$

which transforms $\nu_{r}^{(0)}$ into the result of the SCBA theory 26 applied to the system of SEs on liquid helium 22 . The total momentum relaxation rate $\nu_{\text {eff }}=\nu_{r}^{(0)}+\nu_{\mathrm{mw}}$.

The results of Eqs. (22) and (23) can be obtained also using the direct definition of the electron current

$$
j_{x}=-e n_{s} \sum_{\mathbf{q}}\left(X^{\prime}-X\right)_{\mathbf{q}}\left(\bar{w}_{r, \mathbf{q}}+\bar{w}_{\mathrm{mw}, \mathbf{q}}^{(+)}+\bar{w}_{\mathrm{mw}, \mathbf{q}}^{(-)}\right) .
$$

Taking into account $\left(X^{\prime}-X\right)_{\mathbf{q}}=q_{y} l_{B}^{2}$, one can find

$$
\begin{gathered}
\sigma_{x x}=\frac{e n_{s} l_{B}^{2}}{E_{\|}} \sum_{\mathbf{q}} q_{y}\left(\bar{w}_{r, \mathbf{q}}+\bar{w}_{\mathrm{mw}, \mathbf{q}}^{(+)}+\bar{w}_{\mathrm{mw}, \mathbf{q}}^{(-)}\right) \simeq \\
\simeq \frac{e^{2} n_{s}}{m_{e}} \frac{\nu_{r}^{(0)}+\nu_{\mathrm{mw}}}{\omega_{c}^{2}},
\end{gathered}
$$

which proves that $\mathbf{F}_{\text {fric }}$ is antiparallel to the current. It should be noted that one cannot disregard the driving field correction $-q_{y} V_{H}$ in the expressions for $\bar{w}_{\mathrm{mw}, \mathbf{q}}^{( \pm)}$and $\bar{w}_{r, \mathbf{q}}$, otherwise scattering probabilities in the direction of the driving force and in the opposite direction would be the same leading to $j_{x}=0$.

SEs on liquid helium form a highly correlated 2D electron system. Even for small densities $n_{e} \sim 10^{6} \mathrm{~cm}^{-2}$, the average energy of Coulomb interaction per particle is much larger than the average kinetic energy $\left(T_{e}\right)$. Under such conditions, the $2 \mathrm{D}$ electron system subjected to a magnetic field can be well described using the concept of the fluctuational electric field 27 . At a finite temperature, for each electron there is an electric field $\mathbf{E}_{f}$ of other electrons caused by fluctuations and directed to an equilibrium position of the electron. Within the cyclotron orbit length, the $\mathbf{E}_{f}$ can be approximately considered as a uniform field, if $B$ is strong enough. Thus, the magnetic field and $\mathbf{E}_{f}$ cause fast rotation of the electron obit center around the equilibrium position. Therefore, such a strongly interacting system can be considered as an ensemble of noninteracting electrons, whose orbit centers move fast in the fluctuational field 22,23 . The distribution of $E_{f}$ is known from numerical calculations 28 .

The fluctuational electric field introduces an additional broadening of maxima of the DSF $\underline{16}, 22$ :

$$
S(q, \Omega)=\frac{2 \sqrt{\pi}}{Z_{\|}} \sum_{n, n^{\prime}} \frac{J_{n, n^{\prime}}^{2}}{\gamma_{n, n^{\prime}}} \exp \left[-\frac{\varepsilon_{n}}{T_{e}}-P_{n, n^{\prime}}(\Omega)\right],
$$

where

$$
P_{n, n^{\prime}}=\frac{\left[\Omega-\left(n^{\prime}-n\right) \omega_{c}-\phi_{n}\right]^{2}}{\gamma_{n, n^{\prime}}^{2}}, \phi_{n}=\frac{\Gamma_{n}^{2}+x_{q} \Gamma_{C}^{2}}{4 T_{e} \hbar},
$$

and

$$
\hbar \gamma_{n, n^{\prime}}=\sqrt{\frac{\Gamma_{n}^{2}+\Gamma_{n^{\prime}}^{2}}{2}+x_{q} \Gamma_{C}^{2}} .
$$

In Eq. (28), defining $P_{n, n^{\prime}}(\Omega)$, we have neglected terms of the order of $\Gamma_{n}^{2} / 8 T_{e}^{2}$ which are very small for the considered system. The Coulomb broadening parameter $\Gamma_{C}=\sqrt{2} e E_{f}^{(0)} l_{B}$ increases with $n_{e}$ and $T_{e}$ because $E_{f}^{(0)} \simeq 3 \sqrt{T_{e}} n_{e}^{3 / 4}$.

The fluctuational field introduces also an additional shift in positions of maxima of the DSF $\phi_{C}=x_{q} \Gamma_{C}^{2} / 4 T_{e} \hbar$ which enters the definition of $\phi_{n}$. This Coulomb shift is restored from the expression for the DSF of the 2D Wigner solid under a strong magnetic field. It preserves the equilibrium property of Eq. (20). The influence of this shift on the intersubband displacement mechanism of MO 16 was recently confirmed in experiments ${ }^{17}$ on SEs above liquid ${ }^{3} \mathrm{He}$.

In Eq. (22), describing $\nu_{\mathrm{mw}}$, the function $\chi_{\mathbf{q}}(\omega)$ is averaged over directions of the ripplon vector $\left\langle\chi_{\mathbf{q}}(\omega) \sin ^{2} \varphi\right\rangle_{\varphi} \equiv \chi_{\operatorname{tr}}(\omega)$. Simple integration yields

$$
\chi_{\operatorname{tr}}(\omega)=\frac{1}{2} \frac{3 \omega_{c}^{2} / \omega^{2}+1}{\left(\omega_{c}^{2} / \omega^{2}-1\right)^{2}} .
$$


For $\omega^{2} \gg 3 \omega_{c}^{2}$, the $\chi_{\operatorname{tr}}(\omega) \rightarrow 1 / 2$ which coincides with $\left\langle\sin ^{2} \varphi\right\rangle_{\varphi}$ entering $\nu_{r}^{(0)}$. Using this notation, the photonassisted scattering correction $\nu_{\mathrm{mw}}$ can be represented in an analytical form

$$
\nu_{\mathrm{mw}}=\lambda_{m w} \chi_{\mathrm{tr}}(\omega) \frac{2 m_{e} T \Lambda^{2}}{\sqrt{\pi} \alpha \hbar^{3} l_{B}^{2}} F_{\omega}(B),
$$

where

$$
\begin{gathered}
F_{\omega}(B)=-\frac{1}{Z_{\|}} \sum_{n, m} e^{-\varepsilon_{n} / T_{e}} \int_{0}^{\infty} d x_{q} x_{q} V_{1,1}^{2}\left(x_{q}\right) J_{n, n+m}^{2} \times \\
\frac{\omega / \omega_{c}-m-\phi_{n} / \omega_{c}}{\tilde{\gamma}_{n, n+m}^{3}} \exp \left[-\left(\frac{\omega / \omega_{c}-m-\phi_{n} / \omega_{c}}{\tilde{\gamma}_{n, n+m}}\right)^{2}\right],
\end{gathered}
$$

$\tilde{\gamma}_{n, n+m}=\gamma_{n, n+m} / \omega_{c}$, and $m=n^{\prime}-n$. The dimensionless electron-ripplon coupling ${ }^{22}$

$$
V_{1,1}\left(x_{q}\right)=x_{q} w_{c}\left(x_{q} / 2 g^{2} l_{B}^{2}\right)+e E_{\perp} l_{B}^{2} / \Lambda
$$

is defined by the function

$$
w_{c}(x)=-\frac{1}{1-x}+\frac{1}{(1-x)^{3 / 2}} \ln \left(\frac{1+\sqrt{1-x}}{\sqrt{x}}\right) .
$$

The $V_{1,1}(x)$ depends also on the pressing electric field $E_{\perp}$, the image potential parameter $\Lambda=$ $e^{2}(\epsilon-1) / 4(\epsilon+1)$ (here $\epsilon$ is the dielectric constant of liquid helium), and on the localization parameter $(g)$ of the SE wave function: $\psi_{1}(z)=2 g^{3 / 2} z \exp (-g z)$.

The absolute value of the function $F_{\omega}(B)$ defined by Eq. (32) increases sharply when $\omega / \omega_{c}$ becomes close to an integer $m=n^{\prime}-n>0$. In the vicinity of this point, $F_{\omega}(B)$ changes its sign because of the factor $\omega / \omega_{c}-m-\phi_{n} / \omega_{c}$. The typical dependence $F_{\omega}(B)$ is shown in Fig. 1 for four different electron densities. We have chosen the MW frequency $\omega / 2 \pi=79 \mathrm{GHz}$ which is typical for SEs above liquid ${ }^{3} \mathrm{He}$. It is obvious that an increase in $n_{e}$ suppresses the amplitude of MO and broadens their shape. As expected, negative values of $F_{\omega}(B)$ and $\nu_{\mathrm{mw}}$ mostly appear at $\omega / \omega_{c}(B)>m$.

\section{DISCUSSION AND CONCLUSIONS}

Negative conductivity effects and ZRS appear when $\nu_{\mathrm{mw}} \gtrsim \nu_{\mathrm{eff}}^{(0)}$. It should be notes that $\chi_{\mathrm{tr}}(\omega)$ is about $1 / 2$, if $\omega$ is substantially larger than $\omega_{c}$. As compared to the result of usual electron-ripplon scattering, the momentum relaxation rate caused by photon-assisted scattering [Eq. (31)] has an important proportionality factor $\lambda_{m w}$, which originates from $V_{m w}^{2} /\left(E_{i}-E_{v}\right)^{2} \sim V_{m w}^{2} / \hbar^{2} \omega^{2}$. We note also the presence of the MW frequency $\omega$ in expression for $F_{\omega}(B)$ given in Eq. (32). For usual scattering, the main contribution into $F_{0}(B)$ comes from terms with $m=0$, when the factor $\omega / \omega_{c}-m-\phi_{n} / \omega_{c} \rightarrow$

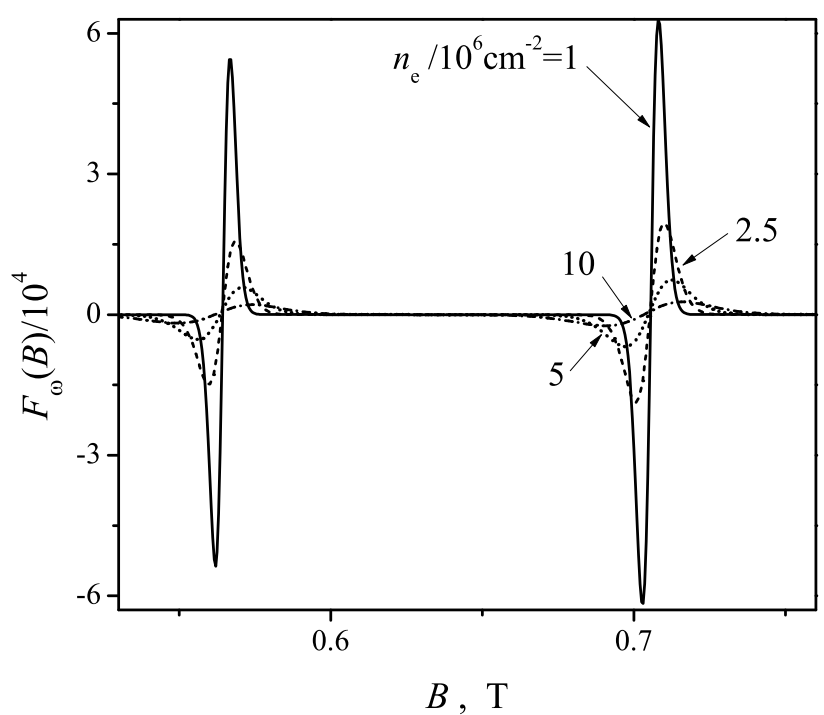

FIG. 1. $\quad F_{\omega}(B)$ calculated near $\omega / \omega_{c}=4$ and 5 for $T=$ $0.2 \mathrm{~K}$ and four electron densities: $n_{e}=1 \cdot 10^{6} \mathrm{~cm}^{-2}$ (solid), $2.5 \cdot 10^{6} \mathrm{~cm}^{-2}$ (dashed), $5 \cdot 10^{6} \mathrm{~cm}^{-2}$ (dotted), $10 \cdot 10^{6} \mathrm{~cm}^{-2}$ (dash-dotted).

$-\phi_{n} / \omega_{c}$ and $F_{0}(B)>0$. In the case of photon-assisted scattering with $\omega>\omega_{c}$, the extrema of $F_{\omega}(B)$ occur at $\left|\omega / \omega_{c}-m\right| \sim \tilde{\gamma}_{n, n+m}$. Therefore, the ratio $F_{\omega}(B) / F_{0}(B) \approx 4 T_{e} / \hbar \gamma_{n, n}$, and we can roughly estimate

$$
\frac{\nu_{\mathrm{mw}}}{\nu_{r}^{(0)}} \sim \lambda_{m w} \frac{4 T_{e}}{\sqrt{\Gamma_{n}^{2}+\Gamma_{C}^{2}}}=\frac{e^{2} E_{m w}^{2} \omega_{c} T_{e}}{m_{e} \hbar \omega^{4} \sqrt{\Gamma_{n}^{2}+\Gamma_{C}^{2}}} .
$$

The $\nu_{\mathrm{mw}} / \nu_{r}^{(0)}$ increases with the MW field amplitude $E_{m w}$ and decreases with the MW frequency.

A more accurate comparison of $\nu_{\mathrm{mw}}$ and $\nu_{r}^{(0)}$ can be given using numerical evaluation of Eq. (32) shown in Fig. 1. Consider typical conditions of the experiment 14 with SEs on liquid ${ }^{3} \mathrm{He}: \omega / 2 \pi \simeq 79 \mathrm{GHz}, T=0.2 \mathrm{~K}$ and $n_{e}=10^{6} \mathrm{~cm}^{-2}$. Under these conditions, the intersubband displacement mechanism 16 leads to giant MO and $\sigma_{x x}<0$ already at a MW field amplitude which corresponds to the Rabi frequency $e E_{m w}|\langle 1|z| 2\rangle| / \hbar=$ $10^{8} \mathrm{~s}^{-1}$. For such a $\mathrm{MW}$ power, $E_{m w} \simeq 0.1 \mathrm{~V} / \mathrm{cm}$, and the calculation based on Eqs. (31) and (32) gives $\delta \nu_{\mathrm{eff}} / \nu_{\mathrm{eff}}^{(0)} \simeq 0.65 \cdot 10^{-5}$, if $B$ is close to $0.7 \mathrm{~T}(m=4)$. This explains why MO caused by photon-assisted scattering were not observed together with MO caused by the intersubband displacement mechanism.

The correction to $\nu_{r}^{(0)}$ caused by the intersubband displacement mechanism $\frac{16}{}$ does not have the proportionality factor $\lambda_{m w}$, because this mechanism does not involve photons. Instead, there is the proportionality factor $\left[\bar{n}_{2}-e^{-\left(\Delta_{2}-\Delta_{1}\right) / T_{e}} \bar{n}_{1}\right]$, where $\bar{n}_{l}=N_{l} / N_{\text {all }}$ is the fractional occupancy of the $l$-th surface subband. This factor is not too small, typically about 0.1 or even larger. There- 
fore, the enhancement factor $4 T_{e} / \hbar \gamma_{n, n^{\prime}}$ noted above can make the amplitude of oscillations very large.

Thus, the main reason why photon-assisted scattering is small in the system of SEs on liquid helium is the parameter $\lambda_{m w}$, which actually does not depend on the nature of scatterers. For a fixed ratio $\omega / \omega_{c}$, it depends only on parameters of the MW field $\left(E_{m w}\right.$ and $\left.\omega\right)$ and on basic parameters of charge carriers (the charge and the effective mass $m_{e}^{*}$ ). According to recent treatments of photon-assisted scattering in semiconductor 2D electron systems $\frac{19}{}$, the effect of MW on the in-plane current $j_{x}$ is characterized by the factor $J_{M}^{2}\left(\sqrt{2 x_{q}} E_{m w} / \tilde{\mathcal{E}}_{\omega}\right)$, where $J_{M}(z)$ is the Bessel function, $M$ is the number of photons assisted in a scattering event, and $\tilde{\mathcal{E}}_{\omega}$ is a characteristic MW field

$$
\tilde{\mathcal{E}}_{\omega}=\frac{\sqrt{2 m_{e}^{*} \omega}\left|\omega_{c}^{2}-\omega^{2}\right| \hbar^{1 / 2}}{e \sqrt{\omega_{c}^{2}+\omega^{2}} \omega_{c}^{1 / 2}}
$$

For $\omega^{2} \gg \omega_{c}^{2}$, the $\tilde{\mathcal{E}}_{\omega} \simeq \sqrt{2} m_{e}^{*} \omega^{2} l_{B} / e$, and the argument of the Bessel function $\sqrt{2 x_{q}} E_{m w} / \tilde{\mathcal{E}}_{\omega} \rightarrow 2 \sqrt{x_{q} \lambda_{m w}}$. In the limit of weak MW fields $E_{m w} \ll \tilde{\mathcal{E}}_{\omega}$, the effect of onephoton assisted scattering is characterized by the small proportionality factor $J_{1}^{2}\left(2 \sqrt{x_{q} \lambda_{m w}}\right) \simeq x_{q} \lambda_{m w}$, which agrees with our calculations.

The main peculiarity of the electron gas in GaAs/AlGaAs, as compared to SEs on liquid helium, is the small effective mass $m_{e}^{*} \simeq 0.067 m_{e}$. Therefore, for the fixed ratio $\omega / \omega_{c}=2$, the Eq. (36) yields: $\tilde{\mathcal{E}}_{\omega} \simeq 74 \mathrm{~V} / \mathrm{cm}$ if $\omega / 2 \pi=79 \mathrm{GHz}$, and $\tilde{\mathcal{E}}_{\omega} \simeq 22 \mathrm{~V} / \mathrm{cm}$ if $\omega / 2 \pi=35.5 \mathrm{GHz}$. It should be noted that these values of $\tilde{\mathcal{E}}_{\omega}$ are much larger than the estimate of the MW field $E_{m w} \approx 0.5 \mathrm{~V} / \mathrm{cm}$ given in Ref. 29 for typical experimental conditions realized in semiconductor systems. For SEs on liquid helium the estimate of the characteristic MW field $\tilde{\mathcal{E}}_{\omega}$ increases only by the factor $1 / \sqrt{0.067} \simeq 3.9$. This factor could be compensated by coordinated reductions in the MW frequency and the cyclotron frequency provided the ratio $\omega_{c} / \omega$ and the MW power are fixed. Therefore, if photon-assisted scattering is the main origin of $\mathrm{MO}$ in GaAs/AlGaAs, it could be potentially observed also in the system of SEs on liquid helium in the low MW frequency range and at highest radiation power.

At this point it is instructive to discuss briefly the inelastic mechanism of MO and ZRS, which is expected to produce larger $\mathrm{MO}$ amplitudes than those of the displacement mechanism. In the inelastic model ${ }^{9}, \mathrm{MO}$ and the negative conductivity effect originate from oscillatory behavior of the electron distribution function $f(\varepsilon)$ entering the conductivity equation for usual scattering which does not involve photons. Oscillatory corrections $\delta f$ to the equilibrium distribution function $f_{F}(\varepsilon)$ are caused by the MW field. The important point of the model is the relationship between $\sigma_{x x}$ and $\partial f(\varepsilon) / \partial \varepsilon$. Sometimes this relationship is referred to Kubo conductivity equations. It should be noted that there the factor $\partial f(\varepsilon) / \partial \varepsilon$ appears from the property of the equilibrium distribution function $f(\varepsilon)[1-f(\varepsilon)]=-T_{e} \partial f / \partial \varepsilon$, which is not valid for an arbitrary function [moreover $f(1-f)>0$ ]. The proper relationship between $\nu_{r}^{(0)}$ and $\partial f / \partial \varepsilon$ can be found considering the nonequilibrium DSF of a $2 \mathrm{D}$ electron gas

$$
\begin{gathered}
S(q, \Omega)=\frac{D}{N_{e} \pi^{2} l_{B}^{2} \hbar} \sum_{n, n^{\prime}} J_{n, n^{\prime}}^{2}\left(x_{q}\right) \int d \varepsilon f(\varepsilon) \times \\
\times[1-f(\varepsilon+\hbar \Omega)] g_{n}(\varepsilon) g_{n^{\prime}}(\varepsilon+\hbar \Omega),
\end{gathered}
$$

where $f(\varepsilon)$ is an arbitrary distribution function and $D$ is the surface area. Now the $S^{\prime}(q, 0) \neq \hbar S(q, 0) / 2 T_{e}$ and one cannot guarantee that $S^{\prime}(q, 0)$ entering Eq. (23) is positive.

Generally, $S^{\prime}(q, \Omega \rightarrow 0)$ consist of the term with the derivative of the factor $1-f(\varepsilon)$ and the term with the derivative of $g_{n^{\prime}}(\varepsilon)$. The second term can be rearranged using integration by parts. Then, using the property $J_{n, n^{\prime}}=J_{n^{\prime}, n}$ one can find

$$
\begin{gathered}
S^{\prime}(q, 0)=\frac{D}{N_{e} 2 \pi^{2} l_{B}^{2}} \sum_{n, n^{\prime}} J_{n, n^{\prime}}^{2}\left(x_{q}\right) \times \\
\times \int d \varepsilon\left[-\frac{\partial f(\varepsilon)}{\partial \varepsilon}\right] g_{n}(\varepsilon) g_{n^{\prime}}(\varepsilon) .
\end{gathered}
$$

This equation together with Eq. (23) establishes the necessary relationship between the momentum relaxation rate and $\partial f(\varepsilon) / \partial \varepsilon$. For interaction with short-range scatterers like vapor atoms, the coupling parameter $C_{\mathbf{q}}^{2}=$ $\hbar^{3} \nu_{0} / 2 m_{e} D$, where $\nu_{0}$ is the collision frequency at $B=0$.

To find corrections to the equilibrium distribution function induced by MW radiation, a sort of kinetic equation was used ${ }^{\underline{9}}$ where the effect of microwaves $\mathrm{St}_{m w}\{f(\varepsilon)\}$ was proportional to $f(\varepsilon \pm \hbar \omega)-f(\varepsilon)$. Since the MW itself cannot cause electron transitions from $\varepsilon$ to $\varepsilon \pm \hbar \omega$ when $\omega / \omega_{c} \geq 2$, we conclude that the main contribution into $\mathrm{St}_{m w}\{f(\varepsilon)\}$ comes from photon-assisted scattering. Though, inelastic mechanism applied to SEs on liquid helium requires a separate investigation, we expect that oscillatory corrections to the equilibrium distribution function will contain the small parameter $\lambda_{m w}$ introduced above. Additionally, electron-electron interaction, which is extremely strong for SEs on liquid helium, should increase substantially the inelastic relaxation rate and reduce the amplitude of MO caused by the inelastic mechanism.

Concluding, for observation of $\mathrm{MO}$ of $\sigma_{x x}$ caused by both of the mechanisms (displacement and inelastic) in the 2D electron system formed on the free surface of liquid helium, it is necessary to use low electron densities and the parameters of the MW field giving a largest value of $\lambda_{m w}$ defined by Eq. (15).

In summary, we have investigated the influence of strong Coulomb forces acting between SEs in liquid helium on photon-assisted scattering and on the displacement mechanism of MW-induced oscillations of magnetoconductivity. Coulomb interaction is shown to suppress 
strongly the amplitude of oscillations and affect their shape. Under conditions of the experiment with SE on liquid helium ${ }^{14}$, the amplitude of MO caused by photonassisted scattering is shown to be very small, which explains why oscillations were not detected for MW frequencies substantially different from the inter-subband resonance frequency. The relationship between the amplitude of $\mathrm{MO}$ and parameters characterized the MW field and the electron system obtained here allows to for- mulate conditions under which photon-assisted scattering could be observed. In order to obtain the same effect as in heterostructures, one need to increase the MW field amplitude by a factor of about 3.9. Therefore, the system of SEs on liquid helium can serve as a model system for testing mechanisms of $\mathrm{MO}$ and ZRS proposed for a $2 \mathrm{D}$ electron gas in heterostructures.

The work was partially supported by a Grant of SFFR and JSPS (F52.2/005).
1 M.A. Zudov, R.R. Du, J.A. Simmons, and J.R. Reno, Phys. Rev. B 64, 201311(R) (2001).

2 R. Mani, J.H. Smet, K. von Klitzing, V. Narayanamurti, W.B. Johnson, and V. Umansky, Nature 420, 646 (2002).

3 M.A. Zudov, R.R. Du, L.N. Pfeiffer, and K.W. West, Phys. Rev. Lett. 90, 046807 (2003).

4 A.C. Durst, S. Sachdev, N. Read, and S.M. Girvin, Phys. Rev. Lett. 91, 086803 (2003).

${ }^{5}$ V. Ryzhii and R. Suris, J. Phys.: Cond. Matt. 15, 6855 (2003).

6 V. Shikin, Pis'ma Zh. Eksp. Teor Fiz. 77, 281 (2003) [JETP Lett. 77, 236 (2003)].

7 A. A. Koulakov and M. E. Raikh, Phys. Rev. B 68, 115324 (2003).

8 V. Ryzhii, A. Chaplik, R. Suris, Pis'ma Zh. Eksp. Teor Fiz. 80, 412 (2004) [JETP Lett. 80, 363 (2004)].

9 I.A. Dmitriev, M.G. Vavilov, I.L. Aleiner, A.D. Mirlin, and D.G. Polyakov, Phys. Rev. B 71, 115316 (2005).

10 J. Inarrea and G. Platero, Phys. Rev. B 76, 073311 (2007).

11 S.A. Mikhailov, Phys. Rev. B 83, 155303 (2011).

12 A.V. Andreev, I.L. Aleiner, and A.J. Millis, Phys. Rev. Lett., 91, 056803 (2003).

13 D. Konstantinov and K. Kono, Phys. Rev. Lett. 103, 266808 (2009).

14 D. Konstantinov and K. Kono, Phys. Rev. Lett. 105, 226801 (2010).

15 Yu.P. Monarkha, Fiz. Nizk. Temp. 37, 108 (2011) [Low Temp. Phys. 37, 90 (2011)]; Yu.P. Monarkha, Fiz. Nizk.
Temp. 37, 829 (2011) [Low Temp. Phys. 37, 655 (2011)].

16 Yu.P. Monarkha, Fiz. Nizk. Temp. 38, 579 (2012) [Low Temp. Phys. 38, 451 (2012)].

17 D. Konstantinov, Yu.P. Monarkha, and K. Kono, Phys. Rev. Lett. 111, 266802 (2013).

18 V. I. Ryzhii, Fiz. Tverd. Tela 11, 2577 (1969) [Sov. Phys. Solid State 11, 2078 (1970)];

19 V. Ryzhii, Phys. Rev. B 68, 193402 (2003).

20 S. I. Dorozhkin, Pisma Zh. Eksp. Teor. Fiz. 77, 681 (2003) [JETP Lett. 77, 577 (2003)].

21 F.G. Bass and I.B. Levinson, Zh. Eksp. Teor. Fiz. 49, 914 (1965).

22 Yu.P. Monarkha and K. Kono, Two-Dimensional Coulomb Liquids and Solids, Springer-Verlag, Berlin Heildelberg (2004).

23 Yu.P. Monarkha, E. Teske, and P. Wyder, Phys. Rep. 370, No. 1, pp. 1-61 (2002).

24 Yu.P. Monarkha, Fiz. Nizk. Temp. 39, 1068 (2013) [Low Temp. Phys. 39, 828 (2013)].

25 R.R. Gerhardts, Surf. Sci. 58, 227 (1976).

26 T. Ando and Y. Uemura, J. Phys. Soc. Jpn. 36, 959 (1974).

27 M.I. Dykman and L.S. Khazan, Zh. Eksp. Teor. Fiz. 77, 1488 (1979) [Sov. Phys. JETP 50, 747 (1979)].

28 C. Fang-Yen, M.I. Dykman, and M.J. Lea, Phys. Rev. B 55, 16272 (1997).

29 P.H. Rivera and P.A. Schulz, Phys. Rev. B 70, 075314 (2004). 